PAWEe Woźny (Wrocław)

\title{
RECURRENCE RELATIONS FOR THE COEFFICIENTS OF EXPANSIONS IN CLASSICAL ORTHOGONAL POLYNOMIALS OF A DISCRETE VARIABLE
}

Abstract. A method is given to find a recurrence relation for the coefficients of the series expansion of a function $f$ with respect to classical orthogonal polynomials of a discrete variable, which follows from a linear difference equation satisfied by $f$.

1. Introduction. Let $f(x)$ be a given function which may be written in the form

$$
f(x)=\sum_{k} a_{k}[f] P_{k}(x),
$$

where $\left\{P_{k}(x)\right\}$ is a system of classical orthogonal polynomials of a discrete variable, i.e. Charlier, Meixner, Krawtchouk or Hahn polynomials (see [1], [6], [13]); in the case of Krawtchouk and Hahn polynomials, which are orthogonal on a finite set, we assume that $f$ is a polynomial.

We are looking for the coefficients of the expansion (1.1). Unfortunately, only in exceptional cases can one give them in explicit form, which often requires the knowledge of the theory of special functions. It is necessary to find another, effective way to calculate $a_{k}[f]$.

The theory given in this paper, described in detail in the author's Master thesis [16], makes it possible to construct a recurrence relation for the coefficients of expansion (1.1) in the form

$$
\sum_{i=0}^{r} A_{i}(k) a_{k+i}[f]=B(k),
$$

where $A_{i}(i=0, \ldots, r)$ and $B$ are functions of $k$. The recurrence relation is

2000 Mathematics Subject Classification: 33C45, 39A70, 42C10, 42A16.

Key words and phrases: Fourier coefficients, recurrence relations, difference equations, orthogonal polynomials of a discrete variable. 
based on a difference equation, satisfied by the function $f(x)$ :

$$
\widetilde{\boldsymbol{P}} f(x) \equiv \boldsymbol{P}^{+} f(x)+\boldsymbol{P}^{-} f(x)=g(x),
$$

where

$$
\begin{gathered}
\boldsymbol{P}^{ \pm} f(x):=\sum_{i=0}^{n^{ \pm}} w_{n^{ \pm}, i}^{ \pm}(x)\left(\boldsymbol{D}^{ \pm}\right)^{i} f(x), \\
\boldsymbol{D}^{+} f(x):=f(x+1)-f(x), \quad \boldsymbol{D}^{-} f(x):=f(x)-f(x-1),
\end{gathered}
$$

the $w_{n^{ \pm}, i}^{ \pm}$are polynomials and the coefficients $a_{k}[g]$ exist and are known. The relation (1.2) may give an explicit formula or, when this is not possible, it enables finding the numerical values of $a_{k}[f]$.

To the author's knowledge, no efficient algorithm has existed to construct a relation (1.2) from (1.3). A solution proposed in [14] has many restrictions and often leads to a recurrence relation for $a_{k}[f]$ of a very high order. Special variants of this problem were considered in [3] and [9].

The method of this work is based on an idea given in [8] (see also [11]). The method can be adapted to the case of $q$-classical orthogonal polynomials ([1], [6], [13]). This was done in [10]. The algorithm from the present paper can also be found in [12], where the cases of (continuous) classical orthogonal polynomials and $q$-classical orthogonal polynomials were also considered.

Many tests show that the proposed solution is optimum in general, which means that the recurrence relation for $a_{k}[f]$ is of the lowest possible order.

In $\S 2$, we settle the notation; it is taken from [10] and [12]. In the next section, we recall the properties of orthogonal polynomials of a discrete variable. As a consequence, in $\S 5$, we give the recurrence relation. We construct difference operators $\mathcal{T}^{ \pm}, \mathcal{L}^{ \pm}$(acting on the variable $k$ ) such that

$$
\mathcal{T}^{ \pm}\left(h_{k} a_{k}\left[\boldsymbol{P}^{ \pm} f\right]\right)=\mathcal{L}^{ \pm}\left(h_{k} a_{k}[f]\right)
$$

(for $h_{k}$, see $\S 6$ ). Next, we find an operator $\mathcal{T}$ with the following property:

$$
\mathcal{T}=\mathfrak{C}^{+} \mathcal{T}^{+}=\mathfrak{C}^{-} \mathcal{T}^{-}
$$

for some operators $\mathrm{C}^{+}$and $\mathcal{C}^{-}$. Applying $\mathcal{T}$ to both sides of

$$
h_{k} a_{k}\left[\boldsymbol{P}^{+} f\right]+h_{k} a_{k}\left[\boldsymbol{P}^{-} f\right]=h_{k} a_{k}[g]
$$

we obtain a recurrence relation for the coefficients $a_{k}[f]$ of the form

$$
\mathcal{L}\left(h_{k} a_{k}[f]\right)=\mathcal{T}\left(h_{k} a_{k}[g]\right),
$$

where

$$
\mathcal{L}:=\mathcal{C}^{+} \mathcal{L}^{+}+\mathcal{C}^{-} \mathcal{L}^{-} .
$$

The algorithm for constructing the recurrence relation was implemented in MAPLE $\mathrm{V}([4],[5])$. The programs can be obtained from http://www. ii.uni.wroc.pl/ pwo. 
2. Notation. Script letter operators $(\mathcal{E}, \mathcal{D}, \ldots)$ will always act on the variable $k$. We define the shift operator $\varepsilon^{m} \pi(k):=\pi(k+m)(m \in \mathbb{Z})$. For simplicity, we write $\mathcal{J}:=\mathcal{E}^{0}$ and $\mathcal{E}:=\mathcal{E}^{1}$.

Bold letter operators $(\boldsymbol{D}, \boldsymbol{U}, \ldots)$ will act on the variable $x$. We set

$$
\boldsymbol{D}^{+}:=\boldsymbol{E}^{+}-\boldsymbol{I}, \quad \boldsymbol{D}^{-}:=\boldsymbol{I}-\boldsymbol{E}^{-},
$$

where $\boldsymbol{I}$ is the identity operator and $\boldsymbol{E}^{ \pm} f(x):=f(x \pm 1)$.

We define the Pochhammer symbol $(a)_{k}$ by

$$
(a)_{k}:= \begin{cases}1 & \text { for } k=0 \\ a(a+1) \cdots(a+k-1) & \text { for } k=1,2, \ldots\end{cases}
$$

The hypergeometric function ${ }_{p} F_{q}$ is given by

$$
{ }_{p} F_{q}\left(\begin{array}{c}
a_{1}, \ldots, a_{p} \\
b_{1}, \ldots, b_{q}
\end{array} \mid x\right):=\sum_{k=0}^{\infty} \frac{\left(a_{1}\right)_{k} \ldots\left(a_{p}\right)_{k}}{\left(b_{1}\right)_{k} \ldots\left(b_{q}\right)_{k}} \frac{x^{k}}{k !} .
$$

3. Properties of classical orthogonal polynomials of a discrete variable. Let $\left\{P_{k}(x)\right\}$ be a system of classical orthogonal polynomials of a discrete variable, i.e. Charlier polynomials $C_{k}(x ; a)$, Meixner polynomials $M_{k}(x ; \beta, c)$, Krawtchouk polynomials $K_{k}(x ; p, N)$ or Hahn polynomials $Q_{k}(x ; \alpha, \beta, N)$ (see $\S 6$, Table 6.1$)$. These polynomials have the following properties ([1], [6], [13]):

1. Orthogonality:

$$
\sum_{x=0}^{B-1} \varrho(x) P_{k}(x) P_{l}(x)=\delta_{k l} h_{k} \quad(k, l=0,1, \ldots),
$$

where $h_{k}>0$ for $k=0,1, \ldots$ and $\delta_{k l}$ is the Kronecker delta. Here $B$ is equal to $+\infty,+\infty, N+1$ and $N$ for Charlier, Meixner, Krawtchouk and Hahn polynomials, respectively. The weight function $\varrho$ satisfies the Pearson equation

$$
\boldsymbol{D}^{+}(\sigma \varrho)=\tau \varrho,
$$

where $\sigma$ and $\tau$ are certain polynomials, associated with the system $\left\{P_{k}(x)\right\}$, of degree at most two and exactly one, respectively.

2. Recurrence relation:

where

$$
x P_{k}(x)=X P_{k}(x)
$$

$$
\mathcal{X}:=\xi_{0}(k) \mathcal{E}^{-1}+\xi_{1}(k) \mathcal{J}+\xi_{2}(k) \mathcal{E} .
$$

3. Difference equation:

$$
\boldsymbol{L}_{k} P_{k}(x) \equiv\left\{\sigma(x) \boldsymbol{D}^{+} \boldsymbol{D}^{-}+\tau(x) \boldsymbol{D}^{+}+\lambda_{k}\right\} P_{k}(x)=0 \quad(k=0,1, \ldots),
$$

where $\lambda_{k}:=-k(k-1) \sigma^{\prime \prime} / 2-k \tau^{\prime}$. 
4. Structure relation:

$$
\sigma^{ \pm}(x) \boldsymbol{D}^{ \pm} P_{k}(x)=\lambda_{k} \mathcal{D}^{ \pm} P_{k}(x) \quad(k=0,1, \ldots),
$$

where

$$
\mathcal{D}^{ \pm}:=\delta_{0}^{ \pm}(k) \mathcal{E}^{-1}+\delta_{1}^{ \pm}(k) \mathcal{J}+\delta_{2}^{ \pm}(k) \mathcal{E}
$$

and $\sigma^{-}:=\sigma, \sigma^{+}:=\sigma+\tau$. The operators $\mathcal{D}^{+}$and $\mathcal{D}^{-}$obey the identity

$$
\mathcal{D}^{-}=\mathcal{D}^{+}+\mathcal{J} \text {. }
$$

5. Difference-recurrence equations:

$$
\frac{\sigma^{ \pm}(x)}{x-\zeta^{ \pm}} Q_{\zeta^{ \pm}}^{ \pm} \lambda_{k}^{-1} \boldsymbol{D}^{ \pm} P_{k}(x)=\mathcal{P}_{\zeta^{ \pm}}^{ \pm} P_{k}(x) \quad(k=0,1, \ldots),
$$

where $\zeta^{ \pm}$is any root of the polynomial $\sigma^{ \pm}$,

$$
\mathcal{P}_{\zeta^{ \pm}}^{ \pm}:=\mathcal{J}+\pi^{ \pm}\left(\zeta^{ \pm} ; k\right) \mathcal{E}, \quad Q_{\zeta^{ \pm}}^{ \pm}:=\vartheta^{ \pm}(k) \mathcal{J}+\omega^{ \pm}\left(\zeta^{ \pm} ; k\right) \mathcal{E}
$$

and $\vartheta^{ \pm}(k):=\xi_{0}(k) / \delta_{0}^{ \pm}(k)$.

The coefficients $\xi_{i}, \delta_{i}^{ \pm}(i=0,1,2), \pi^{ \pm}, \omega^{ \pm}$, weight function $\varrho$, polynomials $\sigma^{ \pm}, \tau$ and $h_{k}, \lambda_{k}$ are given in $\S 6$ (see Tables 6.2 and 6.4).

Using (3.3) we obtain

$$
\left(\mathcal{D}^{+}\right)^{i}\left(\mathcal{D}^{-}\right)^{j}=\left(\mathcal{D}^{-}\right)^{j}\left(\mathcal{D}^{+}\right)^{i} \quad(i, j \in \mathbb{N}) .
$$

4. Fourier coefficients. Assume the function $f(x)$ can be expanded in a series (1.1). Then the Fourier coefficients $a_{k}[f]$ of $f$ are given by

$$
a_{k}[f]=\frac{1}{h_{k}} \sum_{x=0}^{B-1} \varrho(x) P_{k}(x) f(x) .
$$

We write, for simplicity of computations,

$$
b_{k}[f]:=h_{k} a_{k}[f] .
$$

We define the difference operators

$$
\boldsymbol{U}^{ \pm}:=\sigma^{ \pm}(x) \boldsymbol{D}^{ \pm}+\tau(x) \boldsymbol{I}, \quad \boldsymbol{Z}_{\zeta^{ \pm}}^{ \pm}:=\left(x-\zeta^{ \pm}\right) \boldsymbol{D}^{ \pm},
$$

where $\zeta^{+}$and $\zeta^{-}$are any roots of the polynomials $\sigma^{+}$and $\sigma^{-}$, respectively.

Lemma 4.1 ([9]). The following identities hold for $i \in \mathbb{N}$ :

$$
b_{k}[p f]=p(X) b_{k}[f], \quad \text { where } p \text { is a polynomial, }
$$

$$
\begin{aligned}
\left(\mathcal{D}^{ \pm}\right)^{i} b_{k}\left[\left(\boldsymbol{D}^{ \pm}\right)^{i} f\right] & =b_{k}[f], \\
b_{k}\left[\left(\boldsymbol{U}^{ \pm}\right)^{i} f\right] & =\left(-\lambda_{k} \mathcal{D}^{\mp}\right)^{i} b_{k}[f] .
\end{aligned}
$$

LEMma 4.2. We have the equality

$$
\mathcal{P}_{\zeta^{ \pm}}^{ \pm} b_{k}\left[\boldsymbol{Z}_{\zeta^{ \pm}}^{ \pm} f\right]=Q_{\zeta^{ \pm}}^{ \pm} b_{k}[f]
$$


Proof. Using (4.1) for $a_{k}[f]$ and (3.4), (3.2) and (4.2), we get

$$
\begin{aligned}
\mathcal{P}_{\zeta^{ \pm}}^{ \pm} b_{k}\left[\boldsymbol{Z}_{\zeta^{ \pm}}^{ \pm} f\right] & =\sum_{x=0}^{B-1} \varrho(x) \boldsymbol{D}^{ \pm} f(x)\left(x-\zeta^{ \pm}\right) \mathcal{P}_{\zeta^{ \pm}}^{ \pm} P_{k}(x) \\
& =Q_{\zeta^{ \pm}}^{ \pm} \lambda_{k}^{-1} \sum_{x=0}^{B-1} \varrho(x) \boldsymbol{D}^{ \pm} f(x) \sigma^{ \pm}(x) \boldsymbol{D}^{ \pm} P_{k}(x) \\
& =Q_{\zeta^{ \pm}}^{ \pm} \mathcal{D}^{ \pm} \sum_{x=0}^{B-1} \varrho(x) \boldsymbol{D}^{ \pm} f(x) P_{k}(x)=\mathcal{Q}_{\zeta^{ \pm}}^{ \pm} \mathcal{D}^{ \pm} b_{k}\left[\boldsymbol{D}^{ \pm} f\right] \\
& =Q_{\zeta^{ \pm}}^{ \pm} b_{k}[f] .
\end{aligned}
$$

REMARK 4.1. Notice that [9] contains results implying two (of the four) identities of the type (4.3) for Hahn polynomials.

5. Results. It is quite easy to deduce a relation (1.2) from equation (1.3) if $\left\{P_{k}(x)\right\}$ is a system of Charlier, Meixner or Krawtchouk polynomials.

The next theorem solves this problem. The proof, based on property (3.5) and Lemma 4.1, may be found in [16].

THEOREM 5.1. Let $\left\{P_{k}(x)\right\}$ be the system of Charlier, Meixner or Krawtchouk polynomials. Let $f$ be a function (in the case of Krawtchouk polynomials, we assume that $f$ is a polynomial) which may be expanded in a series (1.1) and satisfies

$$
\widetilde{\boldsymbol{P}} f(x) \equiv \boldsymbol{P}^{+} f(x)+\boldsymbol{P}^{-} f(x)=g(x),
$$

where

$$
\boldsymbol{P}^{ \pm} f(x):=\sum_{i=0}^{n^{ \pm}} \boldsymbol{Q}_{i}^{ \pm}\left(z_{i}^{ \pm}(x) f(x)\right),
$$

the operators $\boldsymbol{Q}_{i}^{ \pm}$are of the form

$$
\boldsymbol{Q}_{i}^{ \pm}:=\left(\boldsymbol{D}^{ \pm}\right)^{r_{i}^{ \pm}}\left(\boldsymbol{U}^{ \pm}\right)^{t_{i}^{ \pm}} \quad\left(r_{i}^{ \pm}, t_{i}^{ \pm} \in \mathbb{N} ; i=0,1, \ldots, n^{ \pm}\right)
$$

and the $z_{i}^{ \pm}$are polynomials. Assume that the functions $\boldsymbol{Q}_{i}^{ \pm}\left(z_{i} f\right)$ and $g$ may be expanded in series (1.1). Then the coefficients $a_{k}[f]$ obey the recurrence relation

$$
\mathcal{L}\left(h_{k} a_{k}[f]\right)=\mathcal{T}\left(h_{k} a_{k}[g]\right)
$$

where

$$
\mathcal{L}:=\mathcal{C}^{+} \mathcal{L}^{+}+\mathcal{C}^{-} \mathcal{L}^{-}, \quad \mathcal{T}:=\left(\mathcal{D}^{+}\right)^{r^{+}}\left(\mathcal{D}^{-}\right)^{r^{-}}
$$

and 


$$
\mathcal{C}^{ \pm}:=\left(\mathcal{D}^{\mp}\right)^{r^{\mp}}, \quad \mathcal{L}^{ \pm}:=\sum_{i=0}^{n^{ \pm}}\left(\mathcal{D}^{ \pm}\right)^{r^{ \pm}-r_{i}^{ \pm}}\left(-\lambda_{k} \mathcal{D}^{\mp}\right)^{t_{i}^{ \pm}} z_{i}^{ \pm}(\mathcal{X}) .
$$

Here $r^{ \pm}:=\max _{0 \leq i \leq n^{ \pm}} r_{i}^{ \pm}$.

To apply Theorem 5.1, we have to transform the operators (1.4) to the special form defined by (5.1) and (5.2). The transformation of $\boldsymbol{P}^{ \pm}$to the special form was described in [16] (see also [12]).

The next case is more complicated: we want to construct a recurrence relation

$$
\sum_{i=0}^{r} A_{i}(k) a_{k+i}[f]=B(k)
$$

for the coefficients $a_{k}[f]$ in the representation of a polynomial $f$ of degree $\leq$ $N-1(N \in \mathbb{N})$ as a linear combination of Hahn polynomials $Q_{0}(x ; \alpha, \beta, N)$, $Q_{1}(x ; \alpha, \beta, N), \ldots, Q_{N-1}(x ; \alpha, \beta, N)$.

Suppose that $f$ satisfies the difference equation

$$
\widetilde{\boldsymbol{P}} f(x) \equiv \boldsymbol{P}^{+} f(x)+\boldsymbol{P}^{-} f(x)=g(x),
$$

where

$$
\boldsymbol{P}^{ \pm} f(x):=\sum_{i=0}^{n^{ \pm}} \boldsymbol{Q}_{i}^{ \pm}\left(z_{i}^{ \pm}(x) f(x)\right),
$$

the $z_{i}^{ \pm}$are polynomials and

$$
\boldsymbol{Q}_{i}^{ \pm}:=\left(\boldsymbol{D}^{ \pm}\right)^{r_{i}}\left(\boldsymbol{Z}_{\zeta_{i}^{ \pm}}^{ \pm}\right)^{s_{i}}\left(\boldsymbol{U}^{ \pm}\right)^{t_{i}} \quad\left(r_{i}, s_{i}, t_{i} \in \mathbb{N} ; \sigma^{ \pm}\left(\zeta_{i}^{ \pm}\right)=0\right)
$$

for $i=0,1, \ldots, n^{ \pm}$.

In the first step we will construct operators $\mathcal{Q}_{i}^{ \pm}, \mathcal{A}_{i}^{ \pm}$such that

$$
\mathcal{Q}_{i}^{ \pm} b_{k}\left[\boldsymbol{Q}_{i}^{ \pm} f\right]=\mathcal{A}_{i}^{ \pm} b_{k}[f] \text {. }
$$

Next, we will find operators $\mathcal{T}^{ \pm}$such that

$$
\mathcal{T}^{ \pm}=\mathcal{C}_{i}^{ \pm} Q_{i}^{ \pm}
$$

for some operators $\mathcal{C}_{i}^{ \pm}$. This will make it possible to define operators $\mathcal{L}^{ \pm}$ such that

$$
\mathcal{T}^{ \pm} b_{k}\left[\boldsymbol{P}^{ \pm} f\right]=\mathcal{L}^{ \pm} b_{k}[f]
$$

In the last phase we will construct operators $\mathcal{T}, \mathfrak{C}^{ \pm}$which satisfy

$$
\mathcal{T}=\mathrm{e}^{+} \mathcal{T}^{+}=\mathrm{e}^{-} \mathcal{T}^{-}
$$

Now, applying the operator $\mathcal{T}$ to both sides of the equation

$$
b_{k}\left[\boldsymbol{P}^{+} f\right]+b_{k}\left[\boldsymbol{P}^{-} f\right]=b_{k}[g]
$$

we obtain a recurrence relation for the coefficients $b_{k}[f]$ of the form

$$
\left\{\mathrm{e}^{+} \mathcal{L}^{+}+\mathrm{e}^{-} \mathcal{L}^{-}\right\} b_{k}[f]=\mathcal{T} b_{k}[g] .
$$


We will describe the construction of these operators in the "plus" version. For simplicity of notation, we will omit the upper index + whenever possible.

We need to generalize Lemma 4.2. Given any root $\zeta$ of the polynomial $\sigma^{+}$, we introduce the sequences of operators $\left\{\mathcal{P}_{\zeta}^{(i)}\right\}_{i \in \mathbb{N}},\left\{Q_{\zeta}^{(i)}\right\}_{i \in \mathbb{N}}$ by

$$
\mathcal{P}_{\zeta}^{(i)}:=\mathcal{J}+\pi_{i}(\zeta ; k) \mathcal{E}, \quad Q_{\zeta}^{(i)}:=\vartheta(k) \mathcal{J}+\omega_{i}(\zeta ; k) \mathcal{E},
$$

where the coefficients $\pi_{i}, \omega_{i}$ are such that

$$
\begin{aligned}
& \mathcal{P}_{\zeta}^{(0)}=\mathcal{P}_{\zeta}, \quad Q_{\zeta}^{(0)}=Q_{\zeta}, \\
& \mathcal{P}_{\zeta}^{(i)} Q_{\zeta}^{(i-1)}=Q_{\zeta}^{(i)} \mathcal{P}_{\zeta}^{(i-1)} \quad(i=1,2, \ldots) .
\end{aligned}
$$

We have

$$
\begin{aligned}
\pi_{0}(\zeta ; k) & =\pi(\zeta ; k), \quad \omega_{0}(\zeta ; k)=\omega(\zeta ; k) \\
\pi_{i}(\zeta ; k) & =\frac{\omega_{i-1}(\zeta ; k)-\vartheta(k) \pi_{i-1}(\zeta ; k)}{\omega_{i-1}(\zeta ; k+1)-\vartheta(k+1) \pi_{i-1}(\zeta ; k+1)} \pi_{i-1}(\zeta ; k+1), \\
\omega_{i}(\zeta ; k) & =\pi_{i}(\zeta ; k) \frac{\omega_{i-1}(\zeta ; k+1)}{\pi_{i-1}(\zeta ; k+1)} \quad(i=1,2, \ldots) .
\end{aligned}
$$

The explicit forms for $\pi_{i}$ and $\omega_{i}$ are given in Table 6.3 (see $\S 6$ ).

Set

$$
\begin{aligned}
\mathcal{S}_{\zeta}^{(i, j)} & := \begin{cases}\mathcal{J} & (i<j), \\
\mathcal{P}_{\zeta}^{(i)} \mathcal{P}_{\zeta}^{(i-1)} \ldots \mathcal{P}_{\zeta}^{(j)} & (i \geq j \geq 0),\end{cases} \\
z_{\zeta}^{(i)} & :=\mathcal{S}_{\zeta}^{(i-1,0)} \quad(i \geq 0), \\
\mathcal{U}_{\zeta}^{(0)} & :=\mathcal{J} \\
\mathcal{U}_{\zeta}^{(i)} & :=\mathcal{Q}_{\zeta}^{(i-1)} Q_{\zeta}^{(i-2)} \ldots \mathcal{Q}_{\zeta}^{(0)} \quad(i \geq 1) .
\end{aligned}
$$

Now, we can prove a lemma which generalizes property (4.3).

Lemma 5.2. For any natural number $i$ we have the identity

$$
z_{\zeta}^{(i)} b_{k}\left[\boldsymbol{Z}_{\zeta}^{i} f\right]=u_{\zeta}^{(i)} b_{k}[f]
$$

Proof. Using (5.11) repeatedly, we find that

$$
\mathcal{S}_{\zeta}^{(i-1,1)} Q_{\zeta}^{(0)}=Q_{\zeta}^{(i-1)} \mathcal{Z}_{\zeta}^{(i-1)} \quad(i \geq 1)
$$

Applying Lemma 4.2 and (5.12) $i$ times, we get

$$
\begin{aligned}
z_{\zeta}^{(i)} b_{k}\left[\boldsymbol{Z}_{\zeta}^{i} f\right] & =\mathcal{S}_{\zeta}^{(i-1,1)} Q_{\zeta}^{(0)} b_{k}\left[\boldsymbol{Z}_{\zeta}^{i-1} f\right]=Q_{\zeta}^{(i-1)} z_{\zeta}^{(i-1)} b_{k}\left[\boldsymbol{Z}_{\zeta}^{i-1} f\right]=\ldots= \\
& =Q_{\zeta}^{(i-1)} \ldots Q_{\zeta}^{(1)} \mathcal{P}_{\zeta}^{(0)} b_{k}\left[\boldsymbol{Z}_{\zeta} f\right]=\mathcal{U}_{\zeta}^{(i)} b_{k}[f] .
\end{aligned}
$$

From Lemmas 4.1 and 5.2, it follows that

$$
2 b_{k}[\boldsymbol{Q} f]=\mathcal{A} b_{k}[f],
$$


where $\boldsymbol{Q}$ is the "plus" operator of the type (5.4) and

$$
\mathcal{Q}:=\mathcal{D}^{r} \mathcal{Z}_{\zeta}^{(s)}, \quad \mathcal{A}:=\left(-\lambda_{k} \mathcal{D}^{-}\right)^{t} \mathfrak{U}_{\zeta}^{(s)} .
$$

To find the operator $\mathcal{T}^{+}$(see (5.5)), we define a sequence of operators $\left\{\mathcal{R}_{\zeta}^{(i)}\right\}_{i \in \mathbb{N}}$, where $\zeta$ is defined above and

$$
\begin{aligned}
\mathcal{R}_{\zeta}^{(i)} & :=\delta_{0}(k) \mathcal{E}^{-1}+\rho_{i}(\zeta ; k) \mathcal{J}, \\
\rho_{0}(\zeta ; k) & :=\frac{\delta_{2}(k)}{\pi_{0}(\zeta ; k)}, \\
\rho_{i}(\zeta ; k) & :=\frac{\pi_{i-1}(\zeta ; k)}{\pi_{i}(\zeta ; k)} \rho_{i-1}(\zeta ; k+1) \quad(i \geq 1) .
\end{aligned}
$$

The coefficients $\rho_{i}$ are given in $\S 6$.

LEMmA 5.3. The following identities hold:

$$
\begin{aligned}
\mathcal{R}_{\zeta}^{(0)} \mathcal{P}_{\zeta}^{(0)} & =\mathcal{D} \\
\mathcal{R}_{\zeta}^{(i)} \mathcal{P}_{\zeta}^{(i)} & =\mathcal{P}_{\zeta}^{(i-1)} \mathcal{R}_{\zeta}^{(i-1)} \quad(i=1,2, \ldots)
\end{aligned}
$$

We define

$$
\begin{aligned}
\mathcal{M}_{\zeta}^{(i, j)} & := \begin{cases}\mathcal{J} & (i>j), \\
\mathcal{R}_{\zeta}^{(i)} \mathcal{R}_{\zeta}^{(i+1)} \ldots \mathcal{R}_{\zeta}^{(j)} & (0 \leq i \leq j),\end{cases} \\
\mathcal{N}_{\zeta}^{(i)} & :=\mathcal{M}_{\zeta}^{(0, i-1)} \quad(i \geq 0) .
\end{aligned}
$$

It is easy to verify that

$$
\begin{gathered}
\mathcal{D}^{i}=\mathcal{N}_{\zeta}^{(i)} z_{\zeta}^{(i)} \\
z_{\zeta}^{(i)} \mathcal{R}_{\zeta}^{(0)}=\mathcal{R}_{\zeta}^{(i)} \mathcal{S}_{\zeta}^{(i, 1)} \\
z_{\zeta}^{(i)} \mathcal{D}^{j}=\mathcal{M}_{\zeta}^{(i, i+j-1)} z_{\zeta}^{(i+j)} \quad(i, j=0,1, \ldots)
\end{gathered}
$$

Now, we can prove the following lemma:

\section{Lemma 5.4. Define}

$$
Q_{1}:=z_{\zeta}^{\left(s_{1}\right)} \mathcal{D}^{r_{1}}, \quad Q_{2}:=Z_{\zeta}^{\left(s_{2}\right)} \mathcal{D}^{r_{2}},
$$

where $s_{i}, r_{i} \in \mathbb{N}(i=1,2)$, and set

$$
r:=\max \left(r_{1}, r_{2}\right) \quad s:=\max \left(s_{1}+r_{1}, s_{2}+r_{2}\right)-r .
$$

Then the operator $\mathcal{T}:=Z_{\zeta}^{(s)} \mathcal{D}^{r}$ satisfies

$$
\mathcal{T}=\mathcal{C}_{1} Q_{1}=\mathcal{C}_{2} Q_{2},
$$

where

$$
\mathcal{C}_{i}:=\mathcal{M}_{\zeta}^{\left(s, s+r-r_{i}-1\right)} \mathcal{S}_{\zeta}^{\left(s+r-r_{i}-1, s_{i}\right)} \quad(i=1,2)
$$


Proof. From (5.17), it follows that

$$
\begin{aligned}
\mathcal{C}_{i} Q_{i} & =\mathcal{M}_{\zeta}^{\left(s, s+r-r_{i}-1\right)} \mathcal{S}_{\zeta}^{\left(s+r-r_{i}-1, s_{i}\right)} \mathcal{Z}_{\zeta}^{\left(s_{i}\right)} \mathcal{D}^{r_{i}} \\
& =\mathcal{M}_{\zeta}^{\left(s, s+r-r_{i}-1\right)} \mathcal{Z}_{\zeta}^{\left(s+r-r_{i}\right)} \mathcal{D}^{r_{i}}=\mathcal{Z}_{\zeta}^{(s)} \mathcal{D}^{r-r_{i}} \mathcal{D}^{r_{i}}=\mathcal{Z}_{\zeta}^{(s)} \mathcal{D}^{r} .
\end{aligned}
$$

Reasoning as in the proof of Lemma 5.4 and making use of equality (5.15) we obtain

Lemma 5.5. Let $\zeta$ and $\zeta_{*}$ be different roots of the polynomial $\sigma^{+}$. Let $s_{i}, r_{i} \in \mathbb{N}(i=1,2)$ be such that $s_{1}+r_{1} \geq s_{2}+r_{2}$. Define

$$
Q_{1}:=z_{\zeta}^{\left(s_{1}\right)} \mathcal{D}^{r_{1}}, \quad Q_{2}:=z_{\zeta_{*}}^{\left(s_{2}\right)} \mathcal{D}^{r_{2}},
$$

and set

$$
r:=\max \left(r_{1}, s_{2}+r_{2}\right), \quad s:=s_{1}+r_{1}-r .
$$

Then the operator $\mathcal{T}:=Z_{\zeta}^{(s)} \mathcal{D}^{r}$ has the decomposition $\mathcal{T}=\mathcal{C}_{1} Q_{1}=\mathcal{C}_{2} Q_{2}$ where

$$
\mathcal{C}_{1}:=\mathcal{M}_{\zeta}^{\left(s, s_{1}-1\right)}, \quad \mathcal{C}_{2}:=\mathcal{Z}_{\zeta}^{(s)} \mathcal{N}_{\zeta_{*}}^{\left(r-r_{2}\right)} \mathcal{S}_{\zeta_{*}}^{\left(r-r_{2}-1, s_{2}\right)}
$$

Thus, to construct the operator $\mathcal{T}^{+}$for given $Q_{1}, \ldots, Q_{n}$, it is enough to use the above two lemmas repeatedly. This may be done efficiently. Notice that if we group the operators $Q_{i}$ with respect to the parameter $\zeta$, then we will use Lemma 5.5 no more than once. This is described below in detail.

Lemma 5.6. Let the operator $\boldsymbol{P}$, acting on a polynomial $f$, be given by

$$
\boldsymbol{P} f(x):=\sum_{i=0}^{n} \boldsymbol{Q}_{i}\left(z_{i}(x) f(x)\right),
$$

where the $z_{i}$ are polynomials and the $\boldsymbol{Q}_{i}$ are "plus" operators of the type (5.4). Define

$$
\Omega:=\{0,1, \ldots, n\}, \quad \Omega_{\eta}:=\left\{i \in \Omega: \zeta_{i}=\eta\right\} \quad\left(\eta \in\left\{\zeta, \zeta_{*}\right\}\right),
$$

where $\zeta$ and $\zeta_{*}$ are different roots of the polynomial $\sigma^{+}$. Let

$$
r_{\eta}:=\max _{i \in \Omega_{\eta}} r_{i}, \quad s_{\eta}:=\max _{i \in \Omega_{\eta}}\left(s_{i}+r_{i}\right)-r_{\eta} \quad\left(\eta \in\left\{\zeta, \zeta_{*}\right\}\right) .
$$

Let

$$
\begin{gathered}
\left(\omega, \omega_{*}\right):=\left\{\begin{array}{cc}
\left(\zeta, \zeta_{*}\right) & \text { if } s_{\zeta}+r_{\zeta} \geq s_{\zeta_{*}}+r_{\zeta_{*}}, \\
\left(\zeta_{*}, \zeta\right) & \text { if } s_{\zeta}+r_{\zeta}<s_{\zeta_{*}}+r_{\zeta_{*}},
\end{array}\right. \\
r:=\max \left(r_{\omega}, s_{\omega_{*}}+r_{\omega_{*}}\right), \quad s:=s_{\omega}+r_{\omega}-r .
\end{gathered}
$$

Define

$$
\begin{aligned}
\mathcal{T}^{+}: & =\mathcal{Z}_{\omega}^{(s)}\left(\mathcal{D}^{-}\right)^{r}, \\
\mathcal{A}_{i}: & =\mathcal{U}_{\zeta_{i}}^{\left(s_{i}\right)}\left(-\lambda_{k} \mathcal{D}^{-}\right)^{t_{i}} \quad(i \in \Omega), \\
\mathcal{B}_{i}: & =\mathcal{M}_{\eta}^{\left(s_{\eta}, s_{\eta}+r_{\eta}-r_{i}-1\right)} \mathcal{S}_{\eta}^{\left(s_{\eta}+r_{\eta}-r_{i}-1, s_{i}\right)} \quad\left(i \in \Omega_{\eta} ; \eta \in\left\{\zeta, \zeta_{*}\right\}\right),
\end{aligned}
$$




$$
\begin{aligned}
& \mathcal{J}_{\omega}:=\mathcal{M}_{\omega}^{\left(s, s_{\omega}-1\right)}, \quad \mathcal{J}_{\omega_{*}}:=\mathcal{Z}_{\omega}^{(s)} \mathcal{N}_{\omega_{*}}^{\left(r-r_{\omega_{*}}\right)} \mathcal{S}_{\omega_{*}}^{\left(r-r_{\omega_{*}}-1, s_{\omega_{*}}\right)}, \\
& \mathcal{C}_{0}:=\mathcal{T}, \quad \mathcal{C}_{i}:= \begin{cases}\mathcal{J}_{\omega} \mathcal{B}_{i} & \left(i \in \Omega_{\omega}\right), \\
\mathcal{J}_{\omega_{*}} \mathcal{B}_{i} & \left(i \in \Omega_{\omega_{*}}\right),\end{cases}
\end{aligned}
$$

Then

$$
\mathcal{T}^{+} b_{k}[\boldsymbol{P} f]=\mathcal{L} b_{k}[f] .
$$

REMARK 5.1. All the operators defined above and all lemmas have their "minus" analogues.

Below, we will give a method of constructing operators $\mathcal{T}, \mathcal{C}^{ \pm}$such that

$$
\mathcal{T}=\mathcal{C}^{+} \mathfrak{T}^{+}=\mathcal{C}^{-} \mathfrak{T}^{-},
$$

where $\mathcal{T}^{+}$and $\mathcal{T}^{-}$are as in Lemma 5.6 and in its "minus" version.

For $\zeta^{+}, \zeta^{-}$being any roots of $\sigma^{+}$and $\sigma^{-}$respectively, we introduce the sequences of operators $\left\{\mathcal{F}_{\zeta^{+}, \zeta^{-}}^{ \pm(i)}\right\}_{i \in \mathbb{N}}$ by

$$
\mathcal{F}_{\zeta^{+}, \zeta^{-}}^{ \pm(i)}:=\varphi^{ \pm}\left(\zeta^{+}, \zeta^{-} ; k\right) \mathcal{E}^{-1}+\varphi_{i}^{ \pm}\left(\zeta^{+}, \zeta^{-} ; k\right) \mathcal{J},
$$

where

$$
\begin{aligned}
\varphi^{ \pm}\left(\zeta^{+}, \zeta^{-} ; k\right) & :=\frac{\delta_{0}^{\mp}(k)}{k \pm \zeta_{*}^{\mp} \mp \zeta^{ \pm}-1}, \quad \varphi_{0}^{ \pm}\left(\zeta^{+}, \zeta^{-} ; k\right):=\frac{\rho_{0}^{\mp}\left(\zeta^{\mp} ; k\right)}{k \pm \zeta_{*}^{\mp} \mp \zeta^{ \pm}}, \\
\varphi_{i}^{ \pm}\left(\zeta^{+}, \zeta^{-} ; k\right) & :=\frac{\pi_{i-1}^{ \pm}\left(\zeta^{ \pm} ; k\right)}{\pi_{i}^{ \pm}\left(\zeta^{ \pm} ; k\right)} \varphi_{i-1}^{ \pm}\left(\zeta^{+}, \zeta^{-} ; k+1\right) \quad(i \geq 1) .
\end{aligned}
$$

Equations for the above coefficients are given in $\S 6$.

Lemma 5.7. The following identities are true:

$$
\begin{aligned}
& \mathcal{F}_{\zeta^{+}, \zeta^{-}}^{+(0)} \mathcal{P}_{\zeta^{+}}^{+(0)}=\mathcal{F}_{\zeta_{*}^{+}, \zeta_{*}^{-}}^{-(0)} \mathcal{P}_{\zeta_{*}^{-}}^{-(0)}, \\
& \mathcal{F}_{\zeta^{+}, \zeta^{-}}^{ \pm(i)} \mathcal{P}_{\zeta^{ \pm}}^{ \pm(i)}=\mathcal{P}_{\zeta^{ \pm}}^{ \pm(i-1)} \mathcal{F}_{\zeta^{+}, \zeta^{-}}^{ \pm(i-1)} \quad(i=1,2, \ldots),
\end{aligned}
$$

where $\zeta_{*}^{+} \neq \zeta^{+}$and $\zeta_{*}^{-} \neq \zeta^{-}$are roots of $\sigma^{+}$and $\sigma^{-}$, respectively.

We introduce the notations

$$
\begin{aligned}
& \mathcal{W}_{\zeta^{+}, \zeta^{-}}^{ \pm(i, j)}:= \begin{cases}\mathcal{J} & (i>j), \\
\mathcal{F}_{\zeta^{+}, \zeta^{-}}^{ \pm(i)} \mathcal{F}_{\zeta^{+}, \zeta^{-}}^{ \pm(i+1)} \ldots \mathcal{F}_{\zeta^{+}, \zeta^{-}}^{ \pm(j)} & (0 \leq i \leq j),\end{cases} \\
& \mathcal{y}_{\zeta^{+}, \zeta^{-}}^{ \pm(i)}:=\mathcal{W}_{\zeta^{+}, \zeta^{-}}^{ \pm(0, i-1)} \quad(i \geq 0), \\
& \mathcal{K}_{\zeta^{+}, \zeta^{-}}^{ \pm}:=\mathcal{F}_{\zeta^{+}, \zeta^{-}}^{ \pm(0)} \mathcal{P}_{\zeta^{ \pm}}^{ \pm(0)} .
\end{aligned}
$$

It follows from (5.27) that

$$
\left(\mathcal{K}_{\zeta^{+}, \zeta^{-}}^{+}\right)^{i}=\left(\mathcal{K}_{\zeta_{*}^{+}, \zeta_{*}^{-}}^{-}\right)^{i} \quad(i \in \mathbb{N})
$$


From (5.28) we see that for any natural numbers $i, j$,

$$
\mathcal{S}_{\zeta^{ \pm}}^{ \pm(i, j)} \mathcal{F}_{\zeta^{+}, \zeta^{-}}^{ \pm(j)}=\mathcal{F}_{\zeta^{+}, \zeta^{-}}^{ \pm(i+1)} \mathcal{S}_{\zeta^{ \pm}}^{ \pm(i+1, j+1)}
$$

Using this for $j=0$, one can prove that

$$
\left(\mathcal{K}_{\zeta^{+}, \zeta^{-}}^{ \pm}\right)^{i}=y_{\zeta^{+}, \zeta^{-}}^{ \pm(i)} z_{\zeta^{ \pm}}^{ \pm(i)}
$$

From (3.3), from the decomposition of the operator $\mathcal{D}^{ \pm}$(which results from (5.13)), and from identity (5.16) or its "minus" version, we have

$$
\begin{aligned}
z_{\zeta^{ \pm}}^{ \pm(i)}\left(\mathcal{D}^{\mp}\right)^{j} & =z_{\zeta^{ \pm}}^{ \pm(i)}\left(\mathcal{D}^{ \pm} \pm \mathcal{J}\right)\left(\mathcal{D}^{ \pm} \pm \mathcal{J}\right)^{j-1} \\
& =z_{\zeta^{ \pm}}^{ \pm(i)}\left(\mathcal{R}_{\zeta^{ \pm}}^{ \pm(0)} \mathcal{P}_{\zeta^{ \pm}}^{ \pm(0)} \pm \mathcal{J}\right)\left(\mathcal{D}^{ \pm} \pm \mathcal{J}\right)^{j-1} \\
& =\left(\mathcal{R}_{\zeta^{ \pm}}^{ \pm(i)} \mathcal{S}_{\zeta^{ \pm}}^{ \pm(i, 1)} \mathcal{P}_{\zeta^{ \pm}}^{ \pm(0)} \pm \mathcal{Z}_{\zeta^{ \pm}}^{ \pm(i)}\right)\left(\mathcal{D}^{ \pm} \pm \mathcal{J}\right)^{j-1} \\
& =\left(\mathcal{R}_{\zeta^{ \pm}}^{ \pm(i)} \mathcal{P}_{\zeta^{ \pm}}^{ \pm(i)} \pm \mathcal{J}\right) z_{\zeta^{ \pm}}^{ \pm(i)}\left(\mathcal{D}^{ \pm} \pm \mathcal{J}\right)^{j-1}=\ldots \\
& =\left(\mathcal{R}_{\zeta^{ \pm}}^{ \pm(i)} \mathcal{P}_{\zeta^{ \pm}}^{ \pm(i)} \pm \mathcal{J}\right)^{j} z_{\zeta^{ \pm}}^{ \pm(i)}
\end{aligned}
$$

Now, we can prove the following lemma:

Lemma 5.8. Let $\zeta^{ \pm}$and $\zeta_{*}^{ \pm}$be as defined above. Set

$$
\mathcal{T}^{+}:=z_{\zeta^{+}}^{+\left(s^{+}\right)}\left(\mathcal{D}^{+}\right)^{r^{+}}, \quad \mathcal{T}^{-}:=z_{\zeta^{-}}^{-\left(s^{-}\right)}\left(\mathcal{D}^{-}\right)^{r^{-}} \quad\left(s^{ \pm}, r^{ \pm} \in \mathbb{N}\right)
$$

Then the operator

$$
\mathcal{T}:=\mathcal{Z}\left(\mathcal{K}_{\zeta^{+}, \zeta_{*}^{+}}^{+}\right)^{s}\left(\mathcal{D}^{-}\right)^{r^{-}}\left(\mathcal{D}^{+}\right)^{r^{+}},
$$

where $s:=\min \left(s^{+}, s^{-}\right)$and

$$
z:= \begin{cases}Z_{\zeta^{+}}^{+\left(s^{+}-s^{-}\right)} & \left(s^{+} \geq s^{-}\right), \\ z_{\zeta^{-}}^{-\left(s^{-}-s^{+}\right)} & \left(s^{+}<s^{-}\right),\end{cases}
$$

has the decomposition $\mathcal{T}=\mathrm{e}^{+} \mathcal{T}^{+}=\mathfrak{C}^{-} \mathcal{T}^{-}$, where

$$
\begin{aligned}
& \mathcal{C}^{+}:= \begin{cases}\mathcal{W}_{\zeta^{+}, \zeta_{*}^{-}}^{+\left(s^{+}, s^{-}, s^{+}-1\right)}\left(\mathcal{R}_{\zeta^{+}}^{+\left(s^{+}\right)} \mathcal{P}_{\zeta^{+}}^{+\left(s^{+}\right)}+\mathcal{J}\right)^{r^{-}} & \left(s^{+} \geq s^{-}\right), \\
Z \mathcal{Y}_{\zeta^{+}, \zeta_{*}^{+}}^{+s^{+}}\left(\mathcal{R}_{\zeta^{+}}^{+\left(s^{+}\right)} \mathcal{P}_{\zeta^{+}}^{+\left(s^{+}\right)}+\mathcal{J}\right)^{r^{-}} & \left(s^{+}<s^{-}\right),\end{cases} \\
& \mathfrak{C}^{-}:= \begin{cases}z y_{\zeta_{*}^{+}, \zeta^{-}}^{-\left(s^{-}\right)}\left(\mathcal{R}_{\zeta^{-}}^{-\left(s^{-}\right)} \mathcal{P}_{\zeta^{-}}^{-\left(s^{-}\right)}-\mathcal{J}\right)^{r^{+}} & \left(s^{+} \geq s^{-}\right), \\
\mathcal{W}_{\zeta^{+}, \zeta^{-}}^{-\left(s^{-}-s^{+}, s^{-}-1\right)}\left(\mathcal{R}_{\zeta^{-}}^{-\left(s^{-}\right)} \mathcal{P}_{\zeta^{-}}^{-\left(s^{-}\right)}-\mathcal{J}\right)^{r^{+}} & \left(s^{+}<s^{-}\right) .\end{cases}
\end{aligned}
$$

Proof. Using (5.30) repeatedly, we can prove

$$
z_{\zeta^{ \pm}}^{ \pm(i)} y_{\zeta^{+}, \zeta^{-}}^{ \pm(j)}=\mathcal{W}_{\zeta^{+}, \zeta^{-}}^{ \pm(i, i+j-1)} \mathcal{S}_{\zeta^{ \pm}}^{ \pm(i+j-1, j)} \quad(i, j \geq 0)
$$

Let $s^{ \pm}, r^{ \pm} \in \mathbb{N}$ with $s^{+} \geq s^{-}$(if $s^{+}<s^{-}$then the proof is similar). Set $z:=z_{\zeta^{+}}^{+\left(s^{+}-s^{-}\right)}$. Since $\mathcal{D}^{+}$and $\mathcal{D}^{-}$commute (see (3.5)), from (5.29) we find 
that

$$
\mathcal{T}=\mathcal{Z}\left(\mathcal{K}_{\zeta^{+}, \zeta_{*}^{+}}^{+}\right)^{s^{-}}\left(\mathcal{D}^{-}\right)^{r^{-}}\left(\mathcal{D}^{+}\right)^{r^{+}}=\mathcal{Z}\left(\mathcal{K}_{\zeta_{*}^{+}, \zeta^{-}}^{-}\right)^{s^{-}}\left(\mathcal{D}^{+}\right)^{r^{+}}\left(\mathcal{D}^{-}\right)^{r^{-}}
$$

From (5.31), we get

$$
\begin{aligned}
\mathcal{T} & =z_{\zeta^{+}}^{+\left(s^{+}-s^{-}\right)} y_{\zeta^{+}, \zeta_{*}^{-}}^{+\left(s^{-}\right)} z_{\zeta^{+}}^{+\left(s^{-}\right)}\left(\mathcal{D}^{-}\right)^{r^{-}}\left(\mathcal{D}^{+}\right)^{r^{+}} \\
& =z_{\zeta^{+}}^{+\left(s^{+}-s^{-}\right)} y_{\zeta_{*}^{+}, \zeta^{-}}^{-\left(s^{-}\right)} z_{\zeta^{-}}^{-\left(s^{-}\right)}\left(\mathcal{D}^{+}\right)^{r^{+}}\left(\mathcal{D}^{-}\right)^{r^{-}}
\end{aligned}
$$

From (5.37), we obtain

$$
\begin{aligned}
\mathcal{T} & =\mathcal{Z}_{\zeta^{+}}^{+\left(s^{+}-s^{-}\right)} y_{\zeta^{+,} \zeta_{*}^{+}}^{+\left(s^{-}\right)} z_{\zeta^{+}}^{+\left(s^{-}\right)}\left(\mathcal{D}^{-}\right)^{r^{-}}\left(\mathcal{D}^{+}\right)^{r^{+}} \\
& =\mathcal{W}_{\zeta^{+}, \zeta_{*}^{+}}^{+\left(s^{+}-s^{-}, s^{+}-1\right)} \mathcal{S}_{\zeta^{+}}^{+\left(s^{+}-1, s^{-}\right)} \mathcal{Z}_{\zeta^{+}}^{+\left(s^{-}\right)}\left(\mathcal{D}^{-}\right)^{r^{-}}\left(\mathcal{D}^{+}\right)^{r^{+}} \\
& =\mathcal{W}_{\zeta^{+}, \zeta_{*}^{+}}^{\left(s^{+}-s^{-}, s^{+}-1\right)} \mathcal{Z}_{\zeta^{+}}^{+\left(s^{+}\right)}\left(\mathcal{D}^{-}\right)^{r^{-}}\left(\mathcal{D}^{+}\right)^{r^{+}}
\end{aligned}
$$

Then (5.32) implies

$$
\begin{aligned}
\mathcal{T} & =\mathcal{W}_{\zeta^{+}, \zeta_{*}^{+}}^{+\left(s^{+}-s^{-}, s^{+}-1\right)} \mathcal{Z}_{\zeta^{+}}^{+\left(s^{+}\right)}\left(\mathcal{D}^{-}\right)^{r^{-}}\left(\mathcal{D}^{+}\right)^{r^{+}} \\
& =\mathcal{W}_{\zeta^{+}, \zeta_{*}^{-}}^{+\left(s^{+}-s^{-}, s^{+}-1\right)}\left(\mathcal{R}_{\zeta^{+}}^{+\left(s^{+}\right)} \mathcal{P}_{\zeta^{+}}^{+\left(s^{+}\right)}+\mathcal{J}\right)^{r^{-}} Z_{\zeta^{+}}^{+\left(s^{+}\right)}\left(\mathcal{D}^{+}\right)^{r^{+}}=\mathcal{C}^{+} \mathcal{T}^{+}
\end{aligned}
$$

and

$$
\begin{aligned}
\mathcal{T} & =z_{\zeta^{+}}^{+\left(s^{+}-s^{-}\right)} y_{\zeta_{*}^{+}, \zeta^{-}}^{-\left(s^{-}\right)} z_{\zeta^{-}}^{-\left(s^{-}\right)}\left(\mathcal{D}^{+}\right)^{r^{+}}\left(\mathcal{D}^{-}\right)^{r^{-}} \\
& =z_{\zeta^{+}}^{+\left(s^{+}-s^{-}\right)} y_{\zeta_{*}^{+}, \zeta^{-}}^{-\left(s^{-}\right)}\left(\mathcal{R}_{\zeta^{-}}^{-\left(s^{-}\right)} \mathcal{P}_{\zeta^{-}}^{-\left(s^{-}\right)}-\mathfrak{J}\right)^{r^{+}} z_{\zeta^{-}}^{-\left(s^{-}\right)}\left(\mathcal{D}^{-}\right)^{r^{-}}=\mathcal{C}^{-} \mathcal{T}^{-}
\end{aligned}
$$

Now, we can prove the main theorem of this section.

THeOREM 5.9. Let $\left\{P_{k}(x)\right\}$ be the system of Hahn polynomials. Let $f$ be a polynomial which satisfies the difference equation

$$
\boldsymbol{P} f(x) \equiv \boldsymbol{P}^{+} f(x)+\boldsymbol{P}^{-} f(x)=g(x),
$$

where

$$
\boldsymbol{P}^{ \pm} f(x):=\sum_{i=0}^{n^{ \pm}}\left(\boldsymbol{D}^{ \pm}\right)^{r_{i}}\left(\boldsymbol{Z}_{\zeta_{i}^{ \pm}}^{ \pm}\right)^{s_{i}}\left(\boldsymbol{U}^{ \pm}\right)^{t_{i}}\left(z_{i}^{ \pm}(x) f(x)\right),
$$

the $z_{i}^{ \pm}$are polynomials, $r_{i}, s_{i}, t_{i} \in \mathbb{N}$, and $\sigma^{ \pm}\left(\zeta_{i}^{ \pm}\right)=0$ for $i=0,1, \ldots, n^{ \pm}$. Let $\mathcal{T}^{ \pm}$and $\mathcal{L}^{ \pm}$be as defined in Lemma 5.6 and its "minus" version applied to $\boldsymbol{P}^{ \pm}$. Let $\mathrm{C}^{ \pm}$be as defined in Lemma 5.8 and its "minus" version applied to $\mathcal{T}^{ \pm}$. Then the coefficients $a_{k}[f]$ obey the recurrence relation

$$
\mathcal{L}\left(h_{k} a_{k}[f]\right)=\mathcal{T}\left(h_{k} a_{k}[f]\right), \text { where } \mathcal{L}:=\mathrm{C}^{+} \mathcal{L}^{+}+\mathrm{C}^{-} \mathcal{L}^{-} .
$$

Proof. Using Lemma 5.6 we find operators $\mathcal{T}^{ \pm}, \mathcal{L}^{ \pm}$such that

$$
\mathcal{T}^{ \pm} b_{k}\left[\boldsymbol{P}^{ \pm} f\right]=\mathcal{L}^{ \pm} b_{k}[f] .
$$


Applying Lemma 5.8 to $\mathcal{T}^{ \pm}$, we construct operators $\mathcal{T}$ and $\mathrm{e}^{ \pm}$such that $\mathcal{T}=\mathrm{e}^{+} \mathcal{T}^{+}=\mathrm{C}^{-} \mathcal{T}^{-}$. Applying $\mathcal{T}$ to both sides of

$$
b_{k}[\boldsymbol{P} f]=b_{k}[g]
$$

we get

$\mathcal{T} b_{k}[\boldsymbol{P} f]=\mathcal{C}^{+} \mathcal{T}^{+} b_{k}\left[\boldsymbol{P}^{+} f\right]+\mathcal{C}^{-} \mathcal{T}^{-} b_{k}\left[\boldsymbol{P}^{-} f\right]=\left\{\mathcal{C}^{+} \mathcal{L}^{+}+\mathcal{C}^{-} \mathcal{T}^{-}\right\} b_{k}[f]=\mathcal{T} b_{k}[g]$.

Finally, because $b_{k}[f]=h_{k} a_{k}[f]$, we have

$$
\left\{\mathrm{C}^{+} \mathcal{L}^{+}+\mathrm{C}^{-} \mathcal{L}^{-}\right\}\left(h_{k} a_{k}[f]\right)=\mathfrak{T}\left(h_{k} a_{k}[g]\right) .
$$

To apply Theorem 5.9, we have to transform the operators $\boldsymbol{P}^{ \pm}$defined by (1.4) to the form (5.38). This process was described in [16] (see also [12]).

EXAMPLE 5.1. Let coefficients $c_{n k}$ be such that

$$
Q_{n}(x ; \eta, \zeta, N)=\sum_{k=0}^{n} c_{n k} Q_{k}(x ; \alpha, \beta, N),
$$

where $Q_{n}$ is the $n$th Hahn polynomial. For $f(x) \equiv Q_{n}(x ; \eta, \zeta, N)$, equation (3.1) has the form

$$
\begin{aligned}
x(N & +\eta-x) \boldsymbol{D}^{+} \boldsymbol{D}^{-} f(x) \\
& +[(\zeta+1)(N-1)-(\vartheta+1) x] \boldsymbol{D}^{+} f(x)+n(n+\vartheta) f(x)=0,
\end{aligned}
$$

where $\vartheta:=\eta+\zeta+1$. Using $\boldsymbol{D}^{+} \boldsymbol{D}^{-}=\boldsymbol{D}^{+}-\boldsymbol{D}^{-}$we obtain

$-(x+\zeta+1)(x-N+1) \boldsymbol{D}^{+} f(x)+n(n+\vartheta) f(x)+x(x-\eta-N) \boldsymbol{D}^{-} f(x)=0$.

Let

$$
\begin{aligned}
& \boldsymbol{P}^{+} f(x):=-(x+\zeta+1)(x-N+1) \boldsymbol{D}^{+} f(x)+n(n+\vartheta) f(x), \\
& \boldsymbol{P}^{-} f(x):=x(x-\eta-N) \boldsymbol{D}^{-} f(x) .
\end{aligned}
$$

1. General case. We can find a special form of the operators $\boldsymbol{P}^{ \pm}$,

$$
\begin{aligned}
& \boldsymbol{P}^{+} f(x)=\boldsymbol{Z}_{N-1}^{+}\left(z_{1}^{+}(x) f(x)\right)+z_{0}^{+}(x) f(x), \\
& \boldsymbol{P}^{-} f(x)=\boldsymbol{Z}_{0}^{-}\left(z_{1}^{-}(x) f(x)\right)+z_{0}^{-}(x) f(x),
\end{aligned}
$$

where

$$
\begin{gathered}
z_{1}^{+}(x):=-x-\zeta, \quad z_{0}^{+}(x):=x+n(n+\vartheta)-N+1 \\
z_{1}^{-}(x):=x-\eta-N+1, \quad z_{0}^{-}(x):=-x
\end{gathered}
$$

Hence

$$
\begin{gathered}
\mathcal{T}^{+}=z_{N-1}^{+(1)}, \quad \mathcal{T}^{-}=z_{0}^{-(1)} \\
\mathcal{L}^{+}=\mathcal{U}_{N-1}^{+(1)} z_{1}^{+}(X)+\mathcal{Z}_{N-1}^{+(1)} z_{0}^{+}(X), \quad \mathcal{L}^{-}=\mathcal{U}_{0}^{-(1)} z_{1}^{-}(X)+z_{0}^{-(1)} z_{0}^{-}(X), \\
\mathcal{T}=\mathcal{K}_{N-1, N+\alpha}^{+}, \quad \mathcal{C}^{+}=y_{N-1, N+\alpha}^{+(1)}, \quad \mathcal{C}^{-}=y_{-\beta-1,0}^{-(1)} .
\end{gathered}
$$

Also, the coefficients $c_{n k}$ satisfy the recurrence relation

$$
\mathcal{L}\left(h_{k} c_{n k}\right)=0
$$


where $\mathcal{L}:=\mathcal{C}^{+} \mathcal{L}^{+}+\mathcal{C}^{-} \mathcal{L}^{-}$. Writing (5.40) in explicit form we have

$$
A_{-1}(k) c_{n, k-1}+A_{0}(k) c_{n k}+A_{1}(k) c_{n, k+1}=0,
$$

where

$$
\begin{aligned}
A_{-1}(k):= & (2 k+\gamma-1)_{4}(2 k+\gamma+1)(n-k+1)(k+n+\vartheta-1), \\
A_{0}(k):= & k(k-N)(2 k+\gamma)_{3}\{(\beta-\alpha+2 \eta-2 \zeta) k(k+\gamma) \\
& +n(\beta-\alpha)(\vartheta+n)+(\gamma+1)(\eta \beta-\zeta \alpha)\} \\
A_{1}(k):= & (k)_{2}(N-k-1)_{2}(k+\alpha+1)(k+\beta+1) \\
& \times(2 k+\gamma-1)(k+n+\gamma+1)(k-n+\gamma-\vartheta+1)
\end{aligned}
$$

and $\gamma:=\alpha+\beta+1, c_{n n}=1, c_{n, n+1}=0$.

2. Case $\eta=\alpha$. We transform $\boldsymbol{P}^{+}$and $\boldsymbol{P}^{-}$to the form

$$
\begin{aligned}
& \boldsymbol{P}^{+} f(x)=\boldsymbol{Z}_{N-1}^{+}\left(z_{1}^{+}(x) f(x)\right)+z_{0}^{+}(x) f(x), \\
& \boldsymbol{P}^{-} f(x)=\boldsymbol{U}^{-}\left(z_{1}^{-}(x) f(x)\right)+z_{0}^{-}(x) f(x),
\end{aligned}
$$

where

$$
\begin{array}{ll}
z_{1}^{+}(x):=-x-\zeta, & z_{0}^{+}(x):=x+n(n+\alpha+\zeta+1)-N+1, \\
z_{1}^{-}(x):=-1, & z_{0}^{-}(x):=(\beta+1)(N-1)-(\gamma+1) x
\end{array}
$$

and $\gamma:=\alpha+\beta+1$. We find that

$$
\begin{gathered}
\mathcal{T}^{+}=\mathcal{Z}_{N-1}^{+}, \quad \mathcal{T}^{-}=\mathcal{J}, \\
\mathcal{L}^{+}=\mathcal{U}_{N-1}^{+(1)} z_{1}^{+}(X)+Z_{N-1}^{+(1)} z_{0}^{+}(X), \quad \mathcal{L}^{-}=-\lambda_{k} \mathcal{D}^{+} z_{1}^{-}(X)+z_{0}^{-}(X), \\
\mathcal{T}=\mathcal{T}^{+}, \quad \mathcal{C}^{+}=\mathcal{J}, \quad \mathcal{C}^{-}=\mathcal{T}^{+} .
\end{gathered}
$$

Thus, the following recurrence relation for $c_{n k}$ holds:

$$
\left\{\mathrm{C}^{+} \mathcal{L}^{+}+\mathrm{C}^{-} \mathcal{L}^{-}\right\}\left(h_{k} c_{n k}\right)=0 .
$$

The scalar form is

$$
\begin{aligned}
(n-k+1)(k+ & n+\alpha+\zeta)(2 k+\gamma-1)_{2} c_{n, k-1} \\
& +k(k+\alpha)(k-N)(k+n+\gamma)(k-n+\beta-\zeta) c_{n k}=0 .
\end{aligned}
$$

We can find an explicit equation for $c_{n k}(k=0,1, \ldots, n)$; because $c_{n n}=1$, we get

$$
c_{n k}=(-1)^{n-k}\left(\begin{array}{l}
n \\
k
\end{array}\right) \frac{(\zeta-\beta)_{n-k}(N-n)_{n-k}(k+\alpha+1)_{n-k}(n+k+\gamma+1)_{n-k}}{(k+n+\zeta+\alpha+1)_{n-k}(2 k+\gamma+1)_{2 n-2 k}} .
$$

3. Case $\zeta=\beta$. We have

$$
\begin{aligned}
& \boldsymbol{P}^{+} f(x)=\boldsymbol{U}^{+}\left(z_{1}^{+}(x) f(x)\right)+z_{0}^{+}(x) f(x), \\
& \boldsymbol{P}^{-} f(x)=\boldsymbol{Z}_{0}^{-}\left(z_{1}^{-}(x) f(x)\right)+z_{0}^{-}(x) f(x),
\end{aligned}
$$




$$
\begin{gathered}
z_{1}^{+}(x):=1, \quad z_{0}^{+}(x):=n(n+\gamma)-(\beta+1)(N-1)+(\gamma+1) x, \\
z_{1}^{-}(x):=x-\eta-N+1, \quad z_{0}^{-}(x):=-x,
\end{gathered}
$$

where $\gamma:=\alpha+\beta+1$ and

$$
\begin{gathered}
\mathcal{T}^{+}=\mathcal{J}, \quad \mathcal{T}^{-}=z_{0}^{-(1)} \\
\mathcal{L}^{+}=\lambda_{k} \mathcal{D}^{-} z_{1}^{+}(X)+z_{0}^{+}(X), \quad \mathcal{L}^{-}=\mathcal{U}_{0}^{-(1)} z_{1}^{-}(X)+z_{0}^{-(1)} z_{0}^{-}(X), \\
\mathcal{T}=\mathcal{T}^{-}, \quad \mathcal{C}^{+}=\mathcal{T}^{-}, \quad \mathcal{C}^{-}=\mathcal{J} .
\end{gathered}
$$

Finally, the coefficients $c_{n k}$ obey

$$
\mathcal{L}\left(h_{k} c_{n k}\right)=0
$$

where $\mathcal{L}:=\mathcal{C}^{+} \mathcal{L}^{+}+\mathcal{C}^{-} \mathcal{L}^{-}$. Making simplifications and writing (5.45) in scalar form, we obtain

$$
\begin{aligned}
(n-k+1)(k+ & n+\beta+\eta)(2 k+\gamma-1)_{2} c_{n, k-1} \\
& +k(N-k)(k+\beta)(k+n+\gamma)(k-n+\alpha-\eta) c_{n k}=0 .
\end{aligned}
$$

We know that $c_{n n}=1$. Therefore, for $k=0,1, \ldots, n$, we have

$$
c_{n k}=\left(\begin{array}{l}
n \\
k
\end{array}\right) \frac{(\eta-\alpha)_{n-k}(N-n)_{n-k}(k+\beta+1)_{n-k}(n+k+\gamma+1)_{n-k}}{(k+n+\eta+\beta+1)_{n-k}(2 k+\gamma+1)_{2 n-2 k}} .
$$

REMARK 5.2. Notice the following facts:

1. This problem in the general case was solved earlier in [9], where equation (5.40) was obtained. The analogues of (5.42)-(5.44) given there include inaccuracies.

2. In [2] and [15], it was proved, that - in any case - the coefficients $c_{n k}$ satisfy recurrences of order 4 and 8 , respectively.

It is possible to estimate the order of the recurrence relation for the coefficients $a_{k}[f]$ which follows from the difference equation (1.3).

THEOREM 5.10. Let operators $\mathcal{T}$ and $\mathcal{L}$ be such that

$$
\mathcal{T} b_{k}[\widetilde{\boldsymbol{P}} f]=\mathcal{L} b_{k}[f]
$$

where $\widetilde{\boldsymbol{P}}$ is defined by (1.3) and (1.4). Then

$$
\operatorname{ord}(\mathcal{L}) \leq \operatorname{ord}(\mathcal{T})+2 \max \left(M^{+}, M^{-}\right)
$$

where

$$
M^{ \pm}:=\max _{0 \leq i \leq n^{ \pm}, w_{n^{ \pm}, i}^{ \pm} \neq 0}\left(\partial w_{n^{ \pm}, i}^{ \pm}-i\right)
$$

and $\partial w$ denotes the degree of the polynomial $w$.

Acknowledgements. I would like to thank Professor S. Lewanowicz for many valuable pieces of advice and comments. 


\section{Tables}

Table 6.1. Hypergeometric series representation of the classical monic orthogonal polynomials of the discrete variable [1], [6], [13]

Family

Hypergeometric series

Hahn

$$
Q_{k}(x ; \alpha, \beta, N)=\frac{(\beta+1)_{k}(1-N)_{k}}{(k+\alpha+\beta+1)_{k}}
$$

$$
\times{ }_{3} F_{2}\left(\begin{array}{c|c}
-k,-x, k+\alpha+\beta+1 & 1 \\
\beta+1,1-N & 1
\end{array}\right)
$$

Meixner

$$
M_{k}(x ; \beta, c)=(\beta)_{k}\left(\frac{c}{c-1}\right)_{2}^{k} F_{1}\left(\begin{array}{c|c}
-k,-x & \left.1-\frac{1}{c}\right)
\end{array}\right.
$$

Krawtchouk $\quad K_{k}(x ; p, N)=(-N){ }_{k} p^{k}\left(\begin{array}{l}N \\ k\end{array}\right){ }_{2} F_{1}\left(\begin{array}{c|c}-k,-x \\ -N\end{array} \mid-\frac{1}{p}\right)$

\begin{tabular}{|c|c|}
\hline & $\begin{array}{c}Q_{k}(x ; \alpha, \beta, N) \\
\alpha, \beta>-1, N \in \mathbb{N}\end{array}$ \\
\hline$\sigma(x) \equiv \sigma^{-}(x)$ & $x(N+\alpha-x)$ \\
\hline$\tau(x)$ & $(\beta+1)(N-1)-(\gamma+1) x$ \\
\hline$\sigma^{+}(x)$ & $(x+\beta+1)(N-1-x)$ \\
\hline$\varrho(x)$ & $\frac{\Gamma(N+\alpha-x) \Gamma(x+\beta+1)}{\Gamma(x+1) \Gamma(N-x)}$ \\
\hline$\lambda_{k}$ & $k(k+\gamma)$ \\
\hline$h_{k}$ & $\frac{k ! \Gamma(k+\alpha+1) \Gamma(k+\beta+1)(2 k+\gamma+1)_{N-k-1}}{(k+\gamma)_{k}(N-k-1) !}$ \\
\hline$\xi_{0}(k)$ & $\frac{k(N-k)(k+\alpha)(k+\beta)(k+\gamma-1)(k+\gamma+N-1)}{(2 k+\gamma-2)_{2}(2 k+\gamma-1)_{2}}$ \\
\hline$\xi_{1}(k)$ & $\frac{\alpha-\beta+2 N-2}{4}+\frac{\left(\beta^{2}-\alpha^{2}\right)(\gamma+2 N-1)}{4(2 k+\gamma-1)(2 k+\gamma+1)}$ \\
\hline$\xi_{2}(k)$ & 1 \\
\hline$\delta_{0}^{+}(k)=\delta_{0}^{-}(k)$ & $\frac{(N-k)(k+\alpha)(k+\beta)(k+\gamma-1)(k+\gamma+N-1)}{(2 k+\gamma-2)_{2}(2 k+\gamma-1)_{2}}$ \\
\hline$\delta_{1}^{+}(k)=\delta_{1}^{-}(k)-1$ & $\frac{N(\alpha-\beta)-2 k(k+\gamma)-(\gamma-\alpha)(\gamma-1)}{(2 k+\gamma-1)(2 k+\gamma+1)}$ \\
\hline$\delta_{2}^{+}(k)=\delta_{2}^{-}(k)$ & $-\frac{1}{k+\gamma}$ \\
\hline
\end{tabular}

Charlier $\quad C_{k}(x ; a)=(-a)^{k}{ }_{2} F_{0}\left(-k,-x \mid-\frac{1}{a}\right)$

Table 6.2. Data for the monic Hahn polynomial [1], [6], [13] 
Table 6.2 (cont.)

\begin{tabular}{|c|c|}
\hline & $\begin{array}{c}Q_{k}(x ; \alpha, \beta, N) \\
\alpha, \beta>-1, N \in \mathbb{N}\end{array}$ \\
\hline \multirow{2}{*}{$\omega^{ \pm}\left(\zeta^{ \pm} ; k\right)$} & $\delta_{1}^{ \pm}(k) \xi_{0}(k) / \delta_{0}^{ \pm}(k)+\zeta^{ \pm}-\xi_{1}(k)$ \\
\hline & $\overline{\xi_{0}(k+1) \delta_{2}^{ \pm}(k+1) / \xi_{2}(k+1)-\delta_{0}^{ \pm}(k+1)}$ \\
\hline$\pi^{ \pm}\left(\zeta^{ \pm} ; k\right)$ & $\delta_{2}^{ \pm}(k+1) \omega^{ \pm}\left(\zeta^{ \pm} ; k\right) / \xi_{2}(k+1)$ \\
\hline
\end{tabular}

Notes: 1 . $\gamma:=\alpha+\beta+1 ; \sigma^{+}\left(\zeta^{+}\right)=0 ; \sigma^{-}\left(\zeta^{-}\right)=0$.

2. The formulae for $\omega^{ \pm}, \pi^{ \pm}$are valid also for the other three families of polynomials.

Table 6.3. Hahn polynomials: Explicit forms of the coefficients $\pi_{i}^{ \pm}\left(\zeta^{ \pm} ; k\right), \omega_{i}^{ \pm}\left(\zeta^{ \pm} ; k\right)$, $\rho_{i}^{ \pm}\left(\zeta^{ \pm} ; k\right)$ and $\varphi_{i}^{ \pm}\left(\zeta^{+}, \zeta^{-} ; k\right)$

\begin{tabular}{cc}
$\pi_{i}^{+}\left(\zeta^{+} ; k\right)$ & $\frac{(2 k+\gamma)_{2}(2 k+\gamma+1)_{2}}{(2 k+\gamma+i+1)_{2}(k+\gamma)\left(k+\gamma+\zeta^{+}+1\right)\left(k-\zeta_{*}^{+}\right)}$ \\
$\pi_{i}^{-}\left(\zeta^{-} ; k\right)$ & $-\frac{(2 k+\gamma)_{2}(2 k+\gamma+1)_{2}}{(2 k+\gamma+i+1)_{2}(k+\gamma)\left(k+\alpha-\zeta^{-}+1\right)\left(k+\beta+\zeta_{*}^{-}+1\right)}$ \\
$\omega_{i}^{ \pm}\left(\zeta^{ \pm} ; k\right)$ & $-\pi_{i}^{ \pm}\left(\zeta^{ \pm} ; k\right)(k+\gamma+i+1)$ \\
$\rho_{i}^{+}\left(\zeta^{+} ; k\right)$ & $-\frac{\left(k+\gamma+i+\zeta^{+}+1\right)\left(k+i-\zeta_{*}^{+}\right)}{(2 k+\gamma+i)_{2}}$ \\
$\rho_{i}^{-}\left(\zeta^{-} ; k\right)$ & $\frac{\left(k+\gamma+i+N-\zeta^{-}\right)\left(k+i-N+\zeta_{*}^{-}+1\right)}{(2 k+\gamma+i)_{2}}$ \\
$\varphi_{i}^{ \pm}\left(\zeta^{+}, \zeta^{-} ; k\right)$ & $\pm \frac{k+\gamma+i+\zeta^{+}-\zeta^{-}+1}{(2 k+\gamma+i)_{2}}$ \\
\hline
\end{tabular}

Note: $\gamma:=\alpha+\beta+1 ; \sigma^{ \pm}\left(\zeta^{ \pm}\right)=\sigma^{ \pm}\left(\zeta_{*}^{ \pm}\right)=0 ; \zeta^{ \pm} \neq \zeta_{*}^{ \pm}$.

Table 6.4. Data for the monic Charlier, Meixner and Krawtchouk polynomials $[1],[6],[13]$

\begin{tabular}{cccc}
\hline & $C_{k}(x ; a)$ & $M_{k}(x ; \beta, c)$ & $K_{k}(x ; p, N)$ \\
& $a>0$ & $0<c<1, \beta>0$ & $0<p<1, N \in \mathbb{N}$ \\
\hline$\sigma(x) \equiv \sigma^{-}(x)$ & $x$ & $x$ & $x$ \\
$\tau(x)$ & $a-x$ & $\beta c+(c-1) x$ & $(1-p)^{-1}(N p-x)$ \\
$\sigma^{+}(x)$ & $a$ & $c(x+\beta)$ & $p(1-p)^{-1}(N-x)$ \\
$\varrho(x)$ & $\frac{e^{-a} a^{x}}{\Gamma(x+1)}$ & $\frac{c^{x} \Gamma(\beta+x)}{\Gamma(x+1) \Gamma(\beta)}$ & $\frac{N ! p^{x}(1-p)^{N-x}}{\Gamma(x+1) \Gamma(N+1-x)}$ \\
$\lambda_{k}$ & $k$ & $(1-c) k$ & $(1-p)^{-1} k$ \\
\hline
\end{tabular}


Table 6.4 (cont.)

\begin{tabular}{cccc}
\hline & $\begin{array}{c}C_{k}(x ; a) \\
a>0\end{array}$ & $\begin{array}{c}M_{k}(x ; \beta, c) \\
0<c<1, \beta>0\end{array}$ & $\begin{array}{c}K_{k}(x ; p, N) \\
0<p<1, N \in \mathbb{N}\end{array}$ \\
$h_{k}$ & $k ! a^{k}$ & $\frac{k !(\beta)_{k} c^{k}}{(1-c)^{\beta+2 k}}$ & $\frac{N ! k !}{(N-k) !} p^{k}(1-p)^{k}$ \\
$\xi_{0}(k)$ & $a k$ & $\frac{c k(k+\beta-1)}{(1-c)^{2}}$ & $p(1-p) k(N-k+1)$ \\
$\xi_{1}(k)$ & $k+a$ & $\frac{(c+1) k+\beta c}{1-c}$ & $k+p(N-2 k)$ \\
$\xi_{2}(k)$ & 1 & $\frac{c(k+\beta-1)}{(1-c)^{2}}$ & $p(1-p)(N-k+1)$ \\
$\delta_{0}^{+}(k)=\delta_{0}^{-}(k)$ & $a$ & $\frac{c}{1-c}$ & $-p$ \\
$\delta_{1}^{+}(k)=\delta_{1}^{-}(k)-1$ & 0 & 0 & 0 \\
$\delta_{2}^{+}(k)=\delta_{2}^{-}(k)$ & 0 & &
\end{tabular}

\section{References}

[1] R. Álvarez-Nodarse, Polinomios hipergeométricos y q-polinomios, Monografías de la Academia de Ciencias de Zaragoza, 2001.

[2] I. Area, E. Godoy, A. Ronveaux and A. Zarzo, Minimal recurrence relations for connection coefficients between classical orthogonal polynomials: Discrete case, J. Comput. Appl. Math. 89 (1998), 309-325.

[3] S. Belmehdi, S. Lewanowicz and A. Ronveaux, Linearization of the product of orthogonal polynomials of a discrete variable, Appl. Math. (Warsaw) 24 (1997), 445455 .

[4] B. W. Char et al., Maple V Language Reference Manual, Springer, New York 1991.

[5] B. W. Char et al., Maple V Library Reference Manual, Springer, New York, 1991.

[6] R. Koekoek and R. F. Swarttouw, The Askey-scheme of hypergeometric orthogonal polynomials and its q-analogue, Delft Univ. of Technology, Report 98-17, Delft, 1998.

[7] J. Letessier, A. Ronveaux and G. Valent, Fourth-order difference equation for the associated Meixner and Charlier polynomials, J. Comput. Appl. Math. 71 (1996), 331-341.

[8] S. Lewanowicz, A new approach to the problem of constructing recurrence relations for the Jacobi coefficients, Zastos. Mat. 21 (1991), 303-326.

[9] - Recurrence relations for the connection coefficients of orthogonal polynomials of a discrete variable, J. Comput. Appl. Math. 76 (1996), 213-229.

[10] - Construction of recurrences for the coefficients of expansions in q-classical orthogonal polynomials, ibid. 153 (2003), 295-309.

[11] - Recurrences for coefficients in series expansions with respect to classical orthogonal polynomials, Appl. Math. (Warsaw) 29 (2002), 97-116.

[12] S. Lewanowicz and P. Woźny, Algorithms for construction of recurrence relations for the coefficients of expansions in series of classical orthogonal polynomials, Inst. Comput. Sci., Univ. of Wrocław, 2001. http://www.ii.uni.wroc.pl/ ${ }^{\text {sle/publ/ }}$ 
[13] A. F. Nikiforov, S. K. Suslov and V. B. Uvarov, Classical Orthogonal Polynomials of a Discrete Variable, Springer, Berlin, 1991.

[14] L. Rebillard and A. Ronveaux, Expansion of multivariate polynomials of univariate orthogonal polynomials: discrete case, J. Comput. Appl. Math. 133 (2001), 567-578.

[15] A. Ronveaux, S. Belmehdi, E. Godoy and A. Zarzo, Recurrence relation approach for connection coefficients. Application to classical discrete orthogonal polynomials, in: D. Levi and P. Winternitz (eds.), Proc. Internat. Workshop on Symmetries and Integrability of Difference Equations, CRM Proc. Lecture Notes 9, Amer. Math. Soc., Providence, RI, 1996, 321-337.

[16] P. Woźny, Recurrence relations for the coefficients of expansions in classical orthogonal polynomials of a discrete variable, Master thesis, Inst. Comput. Sci., Univ. of Wrocław, 2001 (in Polish).

Institute of Computer Science

University of Wrocław

Przesmyckiego 20

51-151 Wrocław, Poland

E-mail: Pawel.Wozny@ii.uni.wroc.pl 